\title{
Switching Motor Control: Toward an Integrated Amplifier Design with Position Feedback
}

\author{
Günter Niemeyer Jonathan Fiene \\ Telerobotics Lab \\ Stanford University \\ \{gunter.niemeyer, jfiene\}@stanford.edu
}

\begin{abstract}
In this paper we investigate the advantages and feasibility of motor control using very fast (MHz) switching in place of traditional amplifiers. We also propose an integrated motion-control architecture based on a third-order motor model to be implemented in digital logic at an equally high rate. A switching controller combines the current and position feedback paths into a single loop. A model-based observer estimates the unsensed motor velocity. When compared to second-order controllers implemented with traditional amplifiers, the proposed design promises increased performance, better efficiency, and improved velocity estimation. Recent advances in semiconductor technology provide the required fast switching and logic with relatively standard components. Preliminary experiments characterized nonlinearities in the motor electrical dynamics and verified good performance at frequencies up to $5 \mathrm{MHz}$.
\end{abstract}

\section{INTRODUCTION}

The fields of robotics, mechatronics, and haptics all rely heavily on electric motors to provide forces and torques to mechanisms with smooth operation and high bandwidth. These motors are powered by amplifiers that generate the input voltage to drive current through the coil of the motor. The amplifiers generally use current sensing and local feedback to ensure command tracking in the presence of motor motion, disturbances, and unmodeled effects within both the amplifier and the motor.

The majority of commercially available feedback amplifiers employ either linear regulation or pulse-width modulation (PWM) to control current. They use a single sensor and operate on the first-order electrical dynamics of the motor. They are usually treated as black boxes that produce current up to a specified bandwidth, transforming the motor into a torque source.

Most motion-control systems then wrap an outer loop around the amplifier, as shown in the schematic of Fig. 1. This servo loop uses position information to compute motor torque, assuming a second-order mechanical system. Separating the amplifier feedback and servo feedback loops simplifies the design but limits performance in numerous ways. Most importantly, (i) it restricts the servo bandwidth to be well below the amplifier bandwidth, (ii) it ignores any knowledge of back-EMF voltage, treating this effect as an electrical disturbance, and (iii) it forces motor velocity to be computed solely from position measurements.

In this paper, we propose a new paradigm for motor control, combining both feedback loops into the integrated architecture shown in Fig. 2. This approach uses two sensors to observe the third-order system and overcome the three limitations identified above. A Kalman filter is included to improve velocity estimation, combining a complete motor model, knowledge of current, back-EMF voltage, and position differentiation. A switching controller either turns on or turns off the motor to achieve the desired behavior.

Switching control is effective for linear and nonlinear systems with uncertainty, but it is often avoided due to the high-frequency content of the control signal. Electric motors, however, are a natural filter that can isolate the system's mechanical elements from harmful vibrations. Indeed, PWM amplifiers rely on this principle. Firing pulses at $20-$ $80 \mathrm{kHz}$, they adjust the pulse width with sub-microsecond accuracy. The use of switching control instead of pulsewidth modulation will require:

1) The ability to switch the motor voltage efficiently at $1 \mathrm{MHz}$ or above.

2) The ability to sense current and position at an equally high frequency.

3) The ability to implement the digital logic of the switching controller at that frequency.

Over the last few years, modern electronics has reached the ability to easily fulfill these requirements. This paper presents very fast switching of motor voltage and verifies that such operation is both feasible and efficient. Indeed, it is possible to switch input voltages at $5 \mathrm{MHz}$ with good torque output and no substantial or unexpected motor losses. The paper also presents an investigation of the nonlinear behavior of the motor and driver electronics. Subsequent studies are underway to sense the resulting current and implement switching feedback.

We revisit existing architectures and discuss their abilities in Section II and then detail the proposed architecture in Section III. After describing the hardware in Section IV, we present results and conclude in Sections V and VI. 


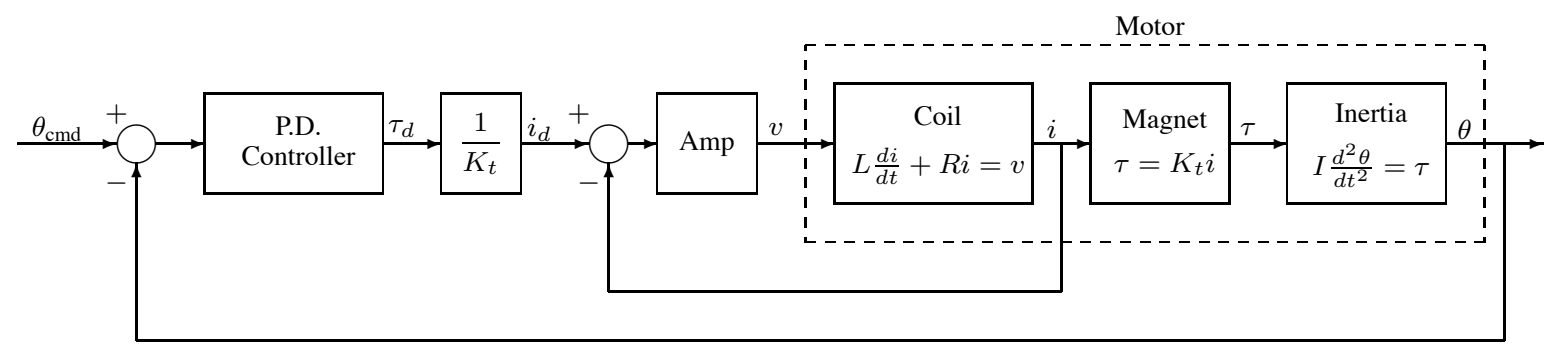

Fig. 1. Block diagram of a typical motion-control architecture using two nested feedback loops.

\section{BACKGROUND}

The dominant existing motion-control architecture completely separates the amplifier and servo feedback loops as depicted in Fig. 1. The inner current feedback loop is implemented inside the amplifier as either linear regulation or pulse-width modulation (PWM). Linear amplifiers tend to have sufficient bandwidth, but may experience crossover distortion when reversing motor polarity and exhibit relatively high amplifier heating. PWM amplifiers remove the excessive amplifier heating because output transistors or MOSFETs are turned fully on or off. Also, the digital nature of a PWM amplifier is attractive to modern designers with single chips providing much of the necessary logic. But switching at $20-80 \mathrm{kHz}$, these amplifiers show a residual current ripple at the switching frequency, introducing electrical noise and possibly significant motor heating. The closed-loop bandwidth of most PWM amplifiers falls in the range of several hundred hertz to a few kilohertz, well below the switching frequency.

Regardless of construction, current feedback usually adds an integral controller to the first-order electrical dynamics of the motor. This provides zero steady-state tracking error between the requested and achieved current, increasing the disturbance rejection. The majority of the disturbance is caused by the back-EMF voltage. Without any knowledge of the motor state or its equations of motion, the amplifiers must treat this very predictable effect as a disturbance.

The outer loop controls motor movement and commonly runs on either a PC or a separate microcontroller. This controller reads position information (typically the discrete output from a digital encoder), computes the desired motor torque, and translates it into a command signal sent to the amplifier. PD or PID control is typical, though other controllers have been designed to improve performance [1]. Built on the idealizing assumption of a second-order system (i.e. assuming perfect current tracking in the amplifiers), this high-level perspective has many inherent performance limits that can be categorized into [2]:

(a) Amplifier bandwidth and roll-off: Designed independently from the amplifier and assuming frequency separation, the servo bandwidth must be significantly below the amplifier bandwidth.

(b) Servo delay: Often implemented on digital computers or microcontrollers, the outer loop has a fixed update rate and a necessary time delay between sensor reading and current command. Again the servo bandwidth must be significantly below this update rate.

(c) Unmodeled dynamics: Unsensed resonances can interact with the amplifier and servo lag to cause instability. To avoid exciting vibrations, the servo bandwidth should be below any unmodeled and unsensed structural resonances or other dynamics.

For a well-designed system, these limits are roughly balanced, and for typical implementations the servo bandwidth falls below $100 \mathrm{~Hz}$.

Most motion commands contain only comparatively lowfrequency components, and tracking is generally not degraded by the limited servo bandwidth. Disturbance rejection, however, depends foremost on the maximum possible feedback gain, which is bounded by the servo bandwidth. In particular, the bandwidth will roughly equal $\sqrt{K_{p} / m}$ where $K_{p}$ is the position feedback gain and $m$ denotes the device inertia. Many designs, especially in haptics and humanfriendly robotics, use lightweight, low-inertia mechanisms to increase safety and reduce force requirements and operator fatigue. These designs lose the ability to properly reject disturbances as the maximum position gain falls below desirable levels.

The velocity feedback gain and hence the ability to apply damping to the outer loop is even further limited. Velocity is rarely measured directly but rather obtained by numerical differentiation of the encoder signal. While encoder signals are typically very clean, the discrete nature and finite resolution of their digital output add uncertainty, especially at low speed and for small movements. When the output signal is differentiated to compute velocity, the discretization noise is amplified, which restricts gains and causes limit cycles. Filtering the velocity signal can help, but it also adds lag, which again limits the closedloop performance. These problems have led to a number of sophisticated algorithms and techniques for estimating velocity from discrete encoder readings [3], [4], [5] and using observers [6], [7]. Meanwhile, an integrated approach incorporating current and back-EMF voltage information into velocity estimates has great potential to improve closedloop feedback performance. 


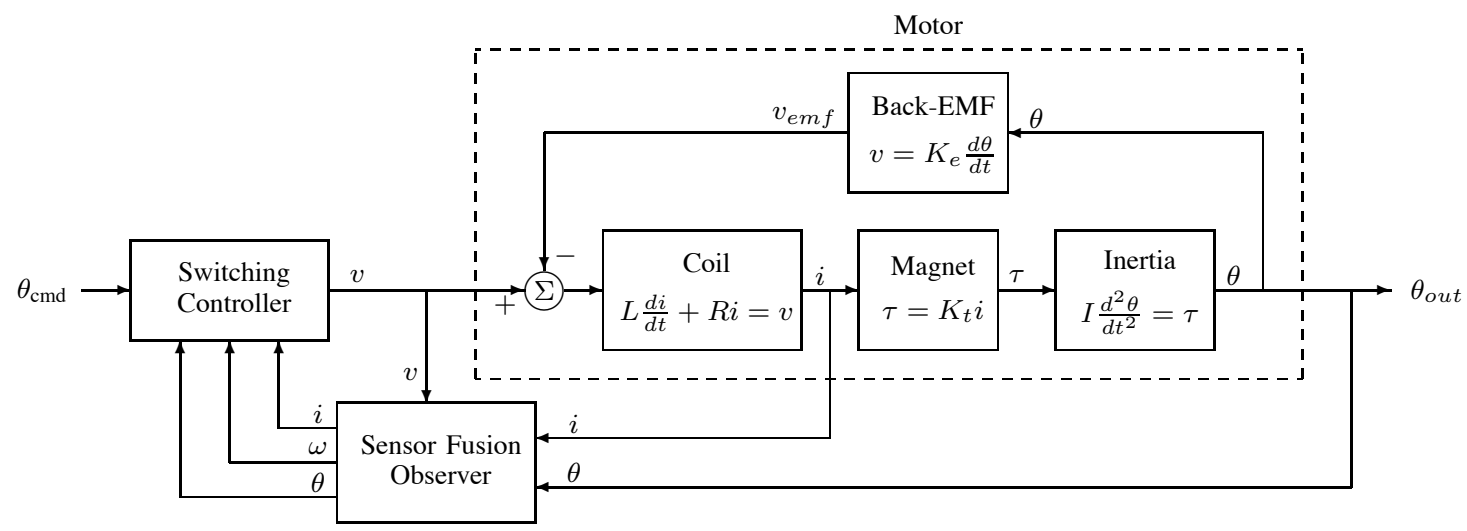

Fig. 2. Block diagram of the proposed motion-control architecture.

\section{Proposed System Architecture}

While the aforementioned loop separation is adequate in many circumstances, it limits operation, especially for high performance systems. We propose replacing the old paradigm of successive loop closure with a new control scheme based on an integrated switching amplifier/controller. Implemented entirely in low-level hardware, this scheme includes both a model-based observer to estimate the unsensed velocity and a switching controller to turn the motor on and off (see Fig. 2).

Designing for a single third-order model has numerous advantages. First, frequency separation is no longer an issue. Second, the model explicitly includes back-EMF, improving tracking and velocity estimation. Third, without a high-level servo loop, there is no servo delay. If the digital logic can be implemented in a single chip, the hardware complexity is comparable to a PWM amplifier. Furthermore, using two sensors to control a third-order system leads to better measurements, especially of velocity, which can be obtained by integrating current as well as by differentiating position. Such model-based estimation can also adapt to motor inductance and resistance, thereby possibly obtaining estimates of motor temperature. Finally, with an appropriately high switching frequency, the current ripple is negligible and motor heating is minimized.

\section{A. Sensor Fusion}

Sensing is a crucial element in any control system. Deriving velocity from both position and current is well posed as the measurements bracket the unsensed state. Position, as the integral of velocity, can be differentiated at low frequency and ensures zero steady state error. On the other hand, current is proportional to torque. If we assume external torque disturbances are of limited frequency, then the motor torque also provides a measure of acceleration at high frequency or for short time durations. Such a strategy can provide high-frequency velocity components, which is perhaps the most significant advantage over traditional approaches.
The motor is generally embedded in a mechanism and connected to the environment only via a transmission. By definition, the inertia and compliance of a transmission filter forces, such that external forces propagating back to the motor should indeed be band-limited. A full model-based observer will generate estimates of these disturbance forces, which could also be made available to a user.

Finally, the full model will include back-EMF effects which further refine the velocity estimate. From the electrical response we can also adapt to the resistance and inductance properties of the motor. This should make an integrated amplifier far more robust to motor selection than traditional amplifiers. And in the limit, slow changes in the resistance may be interpreted as thermal effects and could provide a warning against overheating.

\section{B. Switching Control}

Switching control (sometimes referred to as bang-bang control) is a simple strategy that switches the actuation only between full on and full off [2]. Consider the very basic third-order system

$$
\dddot{x}=f(x, \dot{x}, \ddot{x})+u
$$

where $u$ and $x$ are the system input and output respectively. Also define a switching surface and the distance to that surface as

$$
s=\ddot{x}+2 \lambda \dot{x}+\lambda^{2}\left(x-x_{d}\right)
$$

where $\lambda$ is a preselected constant and $x_{d}$ is a command. Then we can directly control the distance to the surface according to

$$
\dot{s}=\dddot{x}+2 \lambda \ddot{x}+\lambda^{2} \dot{x}=f(x, \dot{x}, \ddot{x})+2 \lambda \ddot{x}+\lambda^{2} \dot{x}+u
$$

If we pick

$$
u= \begin{cases}-u_{\max } & \text { if } s>0 \\ +u_{\max } & \text { if } s<0\end{cases}
$$

and observe that

$$
u_{\max }>\left|f(x, \dot{x}, \ddot{x})+2 \lambda \ddot{x}+\lambda^{2} \dot{x}\right|
$$


then the state of the system will have to converge to the surface (forcing $s \rightarrow 0$ ). From the definition (2) of the surface as well as selection of the parameter $\lambda$, we can verify the convergence of $x$ to $x_{d}$.

For a first-order system, this controller turns the actuation full positive if the error is negative and full negative if the error is positive. In general, switching control performs well, even for nonlinear systems with uncertainty. However, it can suffer from chattering as the actuation is continually reversed. Most systems do not support such operation, with the exception of electrical systems where vibrations do not fatigue the system. In our application, the motor's inductance will filter out this chattering behavior before it reaches any mechanical elements.

Modeling the motor as a third-order system, the three states are the motor position, velocity and current (proportional to torque). The second-order switching surface defines the desired relation between these states. It may be selected to servo the motor to a particular position, hold a particular force, or implement a particular impedance relationship. The benefits of this controller (simplicity, efficiency, performance) make it well suited to driving an electric motor, and continual improvements in semiconductor technology over the past decade have also made it feasible with standard components.

\section{Current Ripple}

Continual switching will induce some ripple in the current and hence in the torque. Using a simplified linear model of the motor as a lumped inductor and resistor, the current response is given by

$$
L \frac{d i}{d t}+R i=v
$$

For this analysis we will assume that steady-state switching will occur in a repeating pattern, i.e. a regular square wave input voltage of amplitude $v_{\max }$ and on/off durations $T_{\text {on }}, T_{\text {off }}$. The theoretical magnitude of the current ripple will be

$$
\Delta i=\frac{v_{\max }}{R} \frac{\left(1-e^{-T_{\mathrm{on}} / \tau}\right)\left(1-e^{-T_{\mathrm{off}} / \tau}\right)}{\left(1-e^{-\left(T_{\mathrm{on}}+T_{\mathrm{off}}\right) / \tau}\right)}
$$

where $\tau=L / R$. This is largest at a $50 \%$ duty cycle, i.e. for $T_{\text {on }}=T / 2$ and $T_{\text {off }}=T / 2$

$$
\Delta i=\frac{v_{\max }}{R} \frac{\left(1-e^{-T / 2 \tau}\right)^{2}}{\left(1-e^{-T / \tau}\right)} \approx \frac{1}{4} \frac{v_{\max }}{R} \frac{T}{\tau}
$$

The final current ripple due to input switching decreases as the frequency of the switching rises and switching times drop below the electrical time constant $\tau$ of the motor. For typical time constants on the order of $100 \mu \mathrm{s}$, switching rates above $1 \mathrm{MHz}$ leave a negligible ripple and subsequently do not add any unnecessary motor heating. Notice that our experimental results show that a linear model of the inductor is not entirely accurate, but we still see the reduction in ripple as frequency increases. The actual frequency of switching will depend entirely on the delay in the electronic circuit, which can easily be as low as $100 \mathrm{~ns}$.

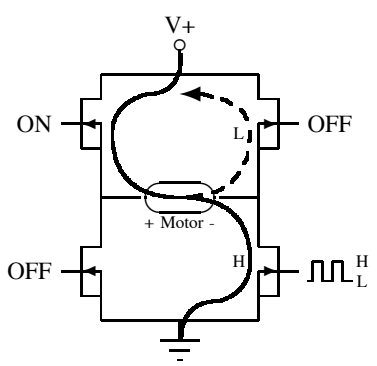

Fig. 3. Amplifier output stage schematic.

\section{Development Plan}

The development and testing plan is organized into three main phases as outlined below. Feasibility has been verified and will be presented in the subsequent sections.

Phase I: Feasibility Study (completed)

1) Motor Modeling: A detailed model of the motor was built for both preliminary simulation and use in the sensor fusion. The transient response characteristics of the motor were of particular interest.

2) High-Frequency Switching: To validate that switching control would be possible, we observed current draw, output torque and heat generation of a DC motor switched up to $5 \mathrm{MHz}$.

Phase II: Sensors and Basic Feedback

1) High-Speed Current Sensing: Selection of an analog to digital converter operating at megahertz speeds will be necessary to close the switching control loop.

2) Basic First-Order Switching Amplifier: A basic firstorder switching amplifier will be built to test the closed-loop behavior of the current regulator.

Phase III: Sensor Fusion and Controller Development

1) Digital Controller Hardware: The controller will be developed using FPGA (Field Programmable Gate Array) or DSP (Digital Signal Processor) technology.

2) Sensor Fusion and Kalman Filter: The sensor fusion algorithm will be developed and implemented as the core of the new architecture.

3) Third-Order Switching Amplifier: The controller will define and regulate the switching surfaces and ultimately require external command inputs.

\section{HARDWARE}

\section{A. Specifications}

Although the proposed system architecture could be utilized in many motion-control applications, the initial design has been concentrated on applications related to humanfriendly and haptic devices. Most of these devices use relatively small motors (1A, 5-15 Watts continuous; 5A, 20-50 Watts peak) operating at less than 30 volts.

\section{B. Preliminary Circuitry}

A fairly simple H-bridge arrangement was selected for the output stage in the initial amplifier design. The H-bridge circuit, as depicted in the schematic of Fig. 3, is composed 


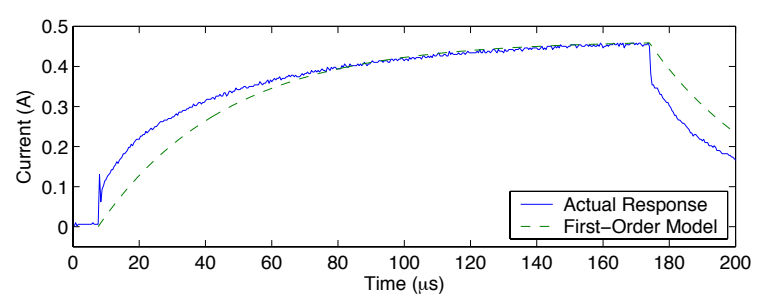

Fig. 4. Comparison of actual motor step response with a first-order model.

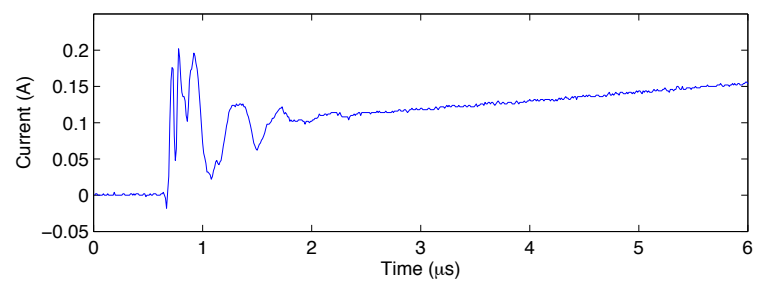

Fig. 5. Close-up of initial step response showing jump and ringing effects.

of four switching elements arranged such that when two diagonally opposing switches are turned on, current flows through the load as shown by the solid line. Changing which pair of switches is on will change the direction of current flow through the load. By using power MOSFETs for the high-side switches, the body diode will prevent inductive voltage spikes by allowing current to continue to flow after the low-side switch is turned off (dashed line in Fig. 3) [8].

$\mathrm{N}$-channel power MOSFETs (IRL520N) were selected for the high-side switches based on their fast reverserecovery time (typically $110 \mathrm{~ns}$ ) and their low on-resistance of less than $0.2 \Omega$. An LM9061N high-side driver IC was chosen to drive the high-side MOSFETs.

The low-side switches need to switch at speeds above the limits of conventional power MOSFETs. A radio frequency power MOSFET driver IC (IXYS DEIC420) with a rated bandwidth over $55 \mathrm{MHz}$ was selected. This driver IC acts very much like a traditional MOSFET, but with very low input capacitance, simplifying the drive circuitry significantly. The driver IC rise and fall times were specified as $4 \mathrm{~ns}$.

\section{Preliminary Test Results}

Two distinct sets of experiments were performed to examine and verify the feasibility of high-speed switching control. First, the motor electrical dynamics were studied by observing the open-loop step response of a stalled motor in the H-bridge circuit. Later experiments considering motor motion and back-EMF effects are reported in [9]. The second set of tests observed torque output and power consumption while switching the open-loop system at frequencies up to $5 \mathrm{MHz}$.

\section{A. Open-Loop Electrical Dynamics and Current Response}

A Maxon brushed RE025-055-38 motor with $830 \mu \mathrm{H}$ inductance and $7.55 \Omega$ coil resistance was placed in series with a $1 \Omega$ current-sense resistor inside the H-bridge circuit. Current response curves were captured using a Tektronix

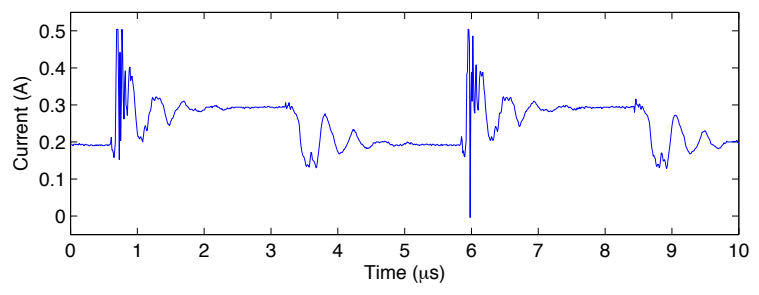

Fig. 6. Open-loop motor current response to a $190 \mathrm{kHz}$ square wave.

460 digitizing oscilloscope and transferred via a GPIB interface to a standard PC. The low-side MOSFET was switched to generate step voltage inputs.

The lumped-parameter linear model given in (6) predicts an exponential rise with time constant $L / R$. With the motor coil placed outside the motor's magnetic field, we indeed see this predicted linear behavior. However, with the motor fully assembled the coil interacts with the magnetic field, and we find significant nonlinear effects. A sample step response and the prediction from the linear model are shown in Fig. 4. This response can be described by three effects. First, at the start of the step, the current exhibits a near-instantaneous jump. The magnitude of the jump is independent of the switching frequency, initial conditions, and even the choice of MOSFET components. The jump scales with applied voltage and decreases with additional external series resistance. Since this jump is not present when the coil is outside of the motor's magnetic field, we believe this effect is due to interactions between the coil and the magnetic field.

The second effect, visible in the close-up of Fig. 5, is high-frequency (approximately $2 \mathrm{MHz}$ ) ringing excited by the step input. This ringing is present with the coil in or out of the magnetic field. It has also been observed when a discrete resistor and inductor were used in place of the motor, showing that this effect is independent of the load. A better understanding of MOSFET properties and careful selection and setup should reduce this effect.

Finally, the current response shows a near-exponential rise to the predicted steady-state value. Slight nonlinearities are observed, though we do not believe they are significant enough to abandon the linear model. When switching at frequencies well above the first-order time constant of $\tau=$ $L / R$, we continue to see the initial jump and high frequency ringing. Fig. 6 shows the step response to a $190 \mathrm{kHz}$ square wave input. Although we have not been able to identify a physical basis for this motor model, it provides an accurate and valuable simulation tool, beneficial to both controller and observer design.

\section{B. High-Speed Switching}

To determine the potential for implementing the switching controller, a Maxim-IC MAX038 IC was used to generate an adjustable high-frequency square wave signal that was used to drive the motor. Measurements of motor output torque (using an ATI Mini-40 6-axis force/torque sensor), average motor current, average motor power and 


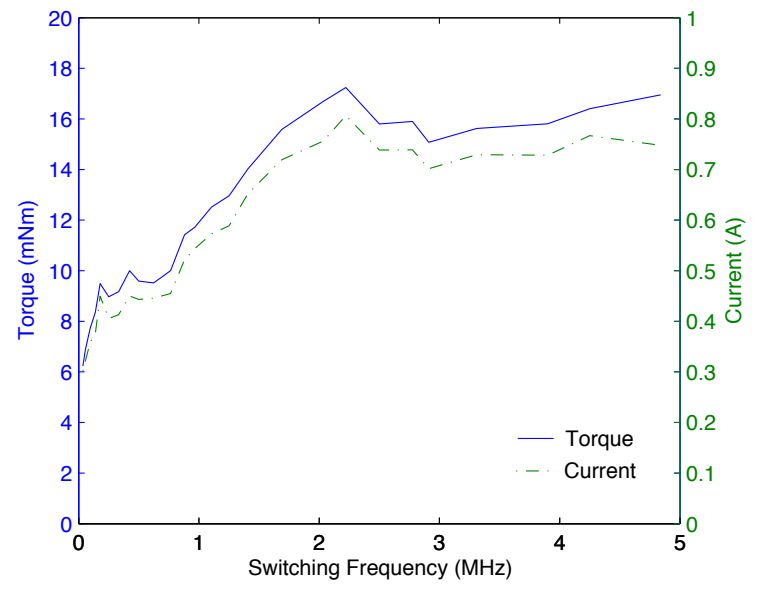

Fig. 7. Variance in motor output torque and motor current.

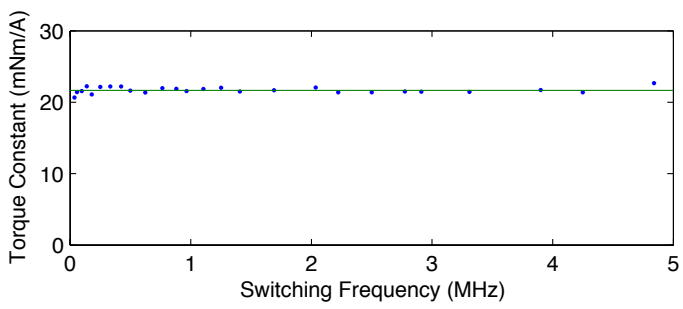

Fig. 8. Torque constant remains constant with switching frequency.

average supply current were taken at discrete frequencies from $20 \mathrm{kHz}$ to over $5 \mathrm{MHz}$. The duty cycle was set to $25 \%$ throughout the experiments, however minor variations (less than 5\%) were observed at different frequencies. The data was compensated accordingly and is shown in Figs. 7-9.

Fig. 7 shows the change in motor output torque and motor current at a fixed duty cycle as a function of switching frequency. It can be seen that both the motor torque and current vary as a function of the switching frequency. Clearly duty cycle, frequency and current do not obey a linear relationship, reiterating the need for closed-loop control. We believe this effect stems from the process of magnetizing and de-magnetizing material within the motor, as well as a possible interaction between the switching input and the MOSFET dynamics observed above.

Motor torque and current, however, follow the same trend, and the torque constant appears unaffected. Fig. 8 shows the observed torque constant as a function of the switching frequency and displays no variation even when switching at speeds well above current PWM rates. Regardless of the duty cycle and frequency at which current is generated, the motor provides the same output torque.

By examining the power consumed in the different elements of the H-bridge and motor, it appears that neither the motor nor the switching elements are experiencing any unexplainable parasitic effects at high frequencies. Fig. 9 shows the motor power as a function of the motor current. The second-order fit $P=I^{2} R$ with $R=6.55 \Omega$ matches the theoretical prediction.

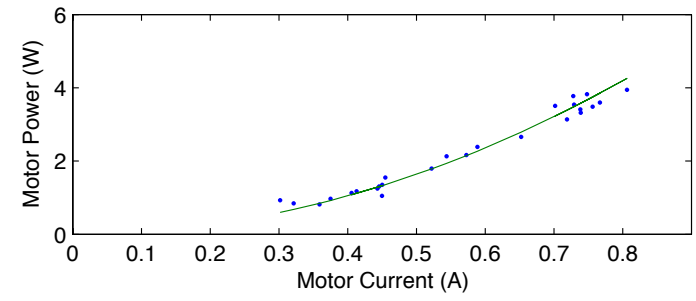

Fig. 9. Power dissipation as a function of motor current.

\section{CONCLUSION}

The use of switching motor control in place of traditional amplifiers shows great promise to improve performance and provide better velocity estimation. This work has demonstrated the ability to switch the input voltage of an electric motor at up to $5 \mathrm{MHz}$, substantially faster than any existing PWM system. Torque generation (as indicated by the torque constant) remains unaffected by switching frequencies, and power consumption follows normal predictions.

We are very encouraged by these early results and are continuing to develop high-speed closed-loop switching control. We hope this approach will enable higher feedback gains, leading to improved performance of haptic and human-friendly devices.

\section{ACKNOWLEDGMENTS}

This material is based upon work supported under a National Science Foundation Graduate Research Fellowship.

\section{REFERENCES}

[1] A. Cavallo and F. Vasca, "Dc motor control with sliding mode switching modulator." in IECON'94 - 20th Annual Conference of IEEE Industrial Electronics, Bologna, Italy, Sept. 1994, pp. 1455-9 vol.3.

[2] J.-J. E. Slotine and W. Li, Applied Nonlinear Control. Englewood Cliffs, New Jersey: Prentice Hall, 1991.

[3] R. Kavanagh, "Signal processing techniques for improved digital tachometry." in ISIE 2002. Proceedings of the 2002 IEEE International Symposium on Industrial Electronics, L'Aquila, Italy, July 2002, pp. 511-17 vol.2.

[4] M. Lemkin, P. Yang, A. Huang, J. Jones, and D. Auslander, "Velocity estimation from widely spaced encoder pulses." in 1995 American Control Conference - ACC'95, Seattle, WA, USA, June 1995, pp. 9981002 vol.1.

[5] G. Liu, "On velocity estimation using position measurements." in 2002 American Control Conference, Anchorage, AK, USA, May 2002, pp. 1115-20 vol.2.

[6] S.-M. Yang and S.-J. Ke, "Performance evaluation of a velocity observer for accurate velocity estimation of servo motor drives." IEEE Transactions on Industry Applications, vol. 36, no. 1, pp. 98 - 104, January 2000.

[7] J. Corres and P. Gil, "High-performance feedforward control of im using speed and disturbance torque observer with noise reduction of shaft encoder." in ISIE 2002. Proceedings of the 2002 IEEE International Symposium on Industrial Electronics, L'Aquila, Italy, July 2002 , pp. $403-8$ vol.2.

[8] P. Horowitz and W. Hill, The Art Of Electronics, Second Edition. Cambridge, United Kingdom: Cambridge University Press, 1989.

[9] J. Fiene and G. Niemeyer, "Switching motor control, an integrated amplifier design for improved velocity estimation and feedback," in Proceedings of the International Conference on Robotics and Automation, Mar. 2004. 